\title{
FORMING EQUATORIAL RINGS AROUND DYING STARS
}

\author{
Muhammad Akashi ${ }^{1}$, Efrat Sabach $^{1}$, Ohad Yogev $^{1}$, \& Noam Soker ${ }^{1}$
}

\begin{abstract}
We suggest that clumpy-dense outflowing equatorial rings around evolved giant stars, such as in supernova 1987A and the Necklace planetary nebula, are formed by bipolar jets that compress gas toward the equatorial plane. The jets are launched from an accretion disk around a stellar companion. Using the FLASH hydrodynamics numerical code we perform 3D numerical simulations, and show that bipolar jets expanding into a dense spherical shell can compress gas toward the equatorial plane and lead to the formation of an expanding equatorial ring. RayleighTaylor instabilities in the interaction region break the ring to clumps. Under the assumption that the same ring-formation mechanism operates in massive stars and in planetary nebulae, we find this mechanism to be more promising for ring formation than mass loss through the second Lagrangian point. The jets account also for the presence of a bipolar nebula accompanying many of the rings.
\end{abstract}

Key words: binaries: general stars: AGB and post-AGB - stars: jets - stars: mass-loss - (ISM:) planetary nebulae: general

\section{INTRODUCTION}

Many nebulae around evolved stars posses a dense equatorial ring. These include both progenitors of core collapse supernovae (CCSNe), e.g., SN 1987A, and lower mass planetary nebulae (PN) and pre-PN, e.g., the Necklace PN (PN G054.2-03.4). There is no consensus yet on the formation mechanism of the equatorial ring and its relation to the bipolar nebula found in all these systems.

The inner (equatorial) ring of 1987A is presently energized by the collision of the SN ejecta, and it can be clearly resolved into many dense clumps (e.g., Larsson et al. 2013, Zanardo et al. 2013). The radius of the ring is $0.21 \mathrm{pc}$ (Plait et al. 1995), and its total ionized mass is $0.06 M_{\odot}$ (Mattila et al. 2010). The center of the ring is offset by $2 \%$ (of its radius) from the SN explosion (Sugerman et al. 2005). The total mass of the circumstellar matter (CSM) nebula of SN 1987A is 1.7M $\odot$ (Sugerman et al. 2005). This implies that the mass in the equatorial ring is a small fraction of the nebular mass (even if the mass in the equatorial ring is several times the ionized mass in the ring). For a recent summary of the CSM properties of SN 1987A see Potter et al. (2014). Smith et al. (2013) and Smith (2007) find the equatorial ring around the B-type supergiant SBW1 and that around the luminous blue variable (LBV) candidate HD 168625, respectively, to be similar in some respects the inner ring of SN 1987A (see also Taylor et al. 2014).

Brandner et al. (1997) point to some similarity between the ring nebula of the blue supergiant Sher 25 and the hourglass shape of the CSM of SN 1987A. For example, the ring in Sher 25 expands at $30 \mathrm{~km} \mathrm{~s}^{-1}$, compared with $\sim 10 \mathrm{~km} \mathrm{~s}^{-1}$ in SN 1987A. Both the ring and the bipolar lobes of Sher 25 were formed in a brief and violent major outburst with a very high mass-loss rate about $6600 \mathrm{yr}$ ago (Brandner et al. 1997).

\footnotetext{
${ }^{3}$ Department of Physics, Technion - Israel Institute of Technology, Haifa 32000 Israel; akashi@physics.technion.ac.il; efrats@physics.technion.ac.il; soker@physics.technion.ac.il.
} 
In SN 1987A, on the other hand, based on dynamical timescales, the inner ring is $\sim 10^{4}$ yr younger than the outer rings. The ionized mass in the hourglass nebula of Sher 25 is $\sim 0.3-0.6 M_{\odot}$, while that in the ring is only $0.01-0.1 M_{\odot}$. Taylor et al. (2014) did not find an interacting or close stellar companion to Sher 25 . In the scenario proposed in the present study a binary companion had entered the envelope of Sher 25 about 6600 yr ago.

The Necklace PN has been studied by Corradi et al. (2011) and its central binary system was further explored by Miszalski et al. (2013). The ring expands with a velocity of $28 \mathrm{~km} \mathrm{~s}^{-1}$, and the total ionized mass of the nebula, most of it in the ring, is $0.06 \pm 0.03 M_{\odot}$. The ring was ejected $\sim 5000$ yr ago and the two opposite polar caps, which were most likely formed by two opposite jets, are $\sim 9000-13,000$ yr old (Corradi et al. 2011; Tocknell et al. 2014). The ring does not seem to posses perfect symmetry around the central star; there seems to be more mass to the north-west direction. The orbital period of the central binary system is 1.16 day, which indicates that the system went through a common envelope (CE) evolution. The total mass in the ring, $\sim 0.06 M_{\odot}$, is much smaller than any mass ejected in the CE process that shrank the binary orbit. A somewhat similar structure of bipolar nebulae and a ring is seen around post-AGB stars (Bujarrabal et al. 2013a), e.g., the Red Rectangle (Cohen et al. 2004; Bujarrabal et al. 2013b). However, in the Red Rectangle the ring is in a Keplerian rotation (Jura et al. 1995), there is an outflowing disk, and the secondary star did not enter a CE phase (van Winckel 2003). This type of systems will be studied in a forthcoming paper.

We note the following common properties to the equatorial rings in the Necklace and most of the equatorial rings of the massive stars with CSM rings, in particular SN 1987A. (1) The general nebular structure composed of the equatorial ring and a bipolar nebula, e.g., a nebula with prominent two outer rings in SN 1987A (Sugerman et al. 2005), and polar clumps (formed by jets) in the Necklace. Other PNe show a clumpy ring in the equatorial plane of a bipolar structure, e.g., NGC 6309 (Rubio et al. 2015). (2) The equatorial ring is broken to many clumps. (3) Displacement from a perfect axisymmetry around the star. This suggests a short interaction time, or interaction in an eccentric orbit. A short interaction time, say few orbital periods or less, suggest instability of the orbital motion. We will examine the possibility of the system being in a Darwin unstable phase during the formation of the ring. (4) The mass in the ring is a small fraction of that in the nebula. (5) Large parts of the polar regions of the nebula, both in SN 1987A and the Necklace, were formed before the ring. However, in Sher 25 the bipolar nebula and ring have been ejected together and during a short time (Brandner et al. 1997). As well, it seems that there are bipolar regions connected to the equatorial ring in SN 1987A (Sugerman et al. 2005) and in SBW1 (Smith et al. 2013). The relation to a bipolar structure and the small mass of the ring suggest that the ring formation comes with the formation of a bipolar nebula. (6) It is commonly accepted that in all systems, PN and massive blue giants, a fast wind, $\sim 500-2000 \mathrm{~km} \mathrm{~s}^{-1}$, was blown shortly after the ejection of the ring.

There is also one significant difference between the systems. In the Necklace the CE process had removed the entire envelope, while in SN 1987A and the other massive stars a massive stellar envelope had survived. Hence, under our assumption of a common formation mechanism, the ring in the Necklace was not formed from the final ejection of the CE.

Let us add here two more systems, the born-again planetary nebulae A30 and A78 (Fang et al. 2014 for a most recent paper and references therein). Both PNe show in their inner region an equatorial ring of knots and faster polar outflows, that Fang et al. (2014) claim to have been ejected in one event in each PN. The polar knots can reach velocities of $\gtrsim 100 \mathrm{~km} \mathrm{~s}^{-1}$. A fast wind $\left(>1000 \mathrm{~km} \mathrm{~s}^{-1}\right)$ blown after the ejection of the ring and polar outflows interacted with the knots (Fang et al. 2014). A late fast wind is common to SN 1987A and the Necklace as well. Such a wind can clean the inner region from low density slowly-moving 
gas, e.g., gas between the dense equators to condense polar regions. There is no indication yet for binary systems at the center of these two PNe.

Another interesting object is the classical nova V959 Mon, for which Chomiuk et al. (2014) argue for equatorial outflow and a faster polar outflow. A clear bi-lobed morphology appears $\sim 100$ days past the detection of $\gamma$-ray emission. Chomiuk et al. (2014) mention that a fast central wind can be focused to the polar directions by the dense equatorial gas. We note that it is quite possible that two opposite jets were launched by the WD few months after outburst, as has been suggested by Retter (2004) for nova V1494 Aql. In this paper we follow Soker \& Rappaport (2000) and raise the possibility that jets compress an equatorial outflow, rather than an equatorial outflow that focuses a polar outflow, in nova V959 Mon and the other objects discussed here.

Many models have been proposed for the formation of equatorial rings, both single-star models, e.g., Chita et al. (2008), and binary models, e.g., Morris \& Podsiadlowski (2009) who studied a merger model for the triple ring system of SN 1987A. We instead take the view that such nebulae are shaped by jets (e.g., Soker 2007), and lobes inflated by the jets can compress an equatorial disk or ring (Soker \& Rappaport 2000. Akashi \& Soker 2008). We will use the common properties to examine a common formation mechanism of the equatorial rings in the systems listed above.

Chita et al. (2008) run a bipolar outflow into a thin shell. Their study has some common features with the mechanism proposed by Soker \& Rappaport (2000) and simulated by Akashi \& Soker (2008). Chita et al. (2008) were aiming at forming several rings outside the equatorial plane. At late times they have no equatorial ring, but only rings above and below the plane. Their equatorial ring is observed only at early times, and it is not formed by compression of gas toward the equatorial plane by jets. The mechanism studied here is based on jets that compress an equatorial outflow, as outlined in Soker \& Rappaport (2000), and should be considered different in many respects from that studied by Chita et al. (2008).

We will differ from Akashi \& Soker (2008) by considering jets-CSM interaction much closer to the binary systems in a process that might also be observed as an Intermediate-Luminosity Optical Transient (ILOT; also termed: Intermediate-Luminosity Red Transient; Red Transient; Red Nova; Luminous Red Nova). ILOTs are eruptive events with a typical decay time of weeks to years and with a peak luminosity between those of novae and supernovae (e.g. Bond et al. 2009; Kashi \& Soker 2010b; Kasliwal et al. 2011, Tylenda et al. 2013). Several studies in recent years pointed to some connections between different ILOTS, e.g, between NGC 300OT and pre-planetary nebulae (PNe) (Prieto et al. 2009), between NGC 300OT and the Great Eruption of $\eta$ Car, (Kashi et al. 2010), and between NGC 300OT and the PN NGC 6302 and the pre-PNs OH231.8+4.2, M1-92 and IRAS 22036+5306 (Soker \& Kashi 2012). Soker \& Kashi (2012) proposed that the lobes of some (but not all) PNs and pre-PNs were formed in a several month-long ILOT event (or several close sub-events). This process was recently simulated by Akashi \& Soker (2013), who also summarize the expected properties of the nebular parts of a PN that are formed by an ILOT event (in general not all nebular components are formed by an ILOT). Boumis \& Meaburn (2013), for example, raised the possibility that the lobes of the PN KjPn 8 were formed in an ILOT event.

In section 2 we examine the possibility that the formation episode of the rings, either by mass loss from the second Lagrangian point $\left(L_{2}\right)$ or by jets, occurred during a relatively short phase of Darwin instability. In section 3 we analyze the mass lose through the second Lagrangian point $\left(L_{2}\right)$ and conclude that it is unlikely by itself to explain the equatorial rings. In section 4 we use a 3D hydrodynamical code to simulate ring formation by a brief jet-launching episode, that might lead also to an ILOT. Our summary is in section 5 . 


\section{DARWIN UNSTABLE PHASE}

We consider a binary system composed of a primary giant star and a more compact secondary star, mainly a main sequence (MS) star. The giant primary star can be a red giant branch (RGB) star, and asymptotic giant branch (AGB) star, e.g., in the case of PNe, or a red supergiant (RSG), e.g., in the case of the progenitor of the SN 1987A nebula. We study processes where the expanding equatorial rings mentioned in section 1 were formed in a relatively short time, i.e., much shorter than the phase of high mass loss rate. The high mass loss rate of AGB and RSG stars can last hundreds to thousands of years, while the brief ring formation episode is assumed to take place within months to tens of years, $\sim 0.03-30 \mathrm{yr}$. We are not considering here the formation of rotating (Keplerian) disks.

We assume that the brief ring formation episode takes place while the system is in an unstable Darwin phase. Before the ring formation episode the secondary star brings the giant envelope to synchronization with the orbital motion (co-rotation), and the the system is Darwin stable, i.e., $I_{B}>3 I_{1}$. Here $I_{B}$ and $I_{1}$ are the moments of inertia of the binary system and the primary star, respectively. Due to further spiraling-in as a result of loss of angular momentum and/or due to the expansion of the giant star, at some stage the system becomes Darwin unstable.

We can constrain the mass of the secondary star under these assumptions when interaction starts. Tidal interaction becomes strong when the primary radius becomes $R_{1} \gtrsim 0.25 a_{0}$, where $a_{0}$ is the orbital separation when tidal interaction starts to dominate the orbital evolution (Soker 1996). We demand that by the time the binary system has spiraled-in to $a_{i} \gtrsim 1.5 R_{1}$ the primary envelope has reached co-rotation (the derivation is not sensitive to the exact value in the range of $\left.a_{i} \simeq 1.2-2 R_{1} \simeq 0.3-0.5 a_{0}\right)$. The moment of inertia of the giant envelope is taken to be $I_{1} \simeq \eta M_{\mathrm{env}} R_{1}^{2}$, where $M_{\mathrm{env}}$ is the convective envelope mass and $R_{1}$ is the giant radius. For AGB stars $\eta \simeq 0.2-0.24$. For the progenitor of SN 1987A during its RSG phase we run a model with an initial mass of $20 M_{\odot}$ using the Modules for Experiments in Stellar Astrophysics (MESA), version 6596 (Paxton et al. 2011). At its RSG phase 20,000 yr before explosion its mass is $16.4 M_{\odot}$, its radius is $R_{1}=1130 R_{\odot}$, the convective envelope mass is $M_{\mathrm{env}}=9.3 M_{\odot}$, and $\eta=0.22$.

We will consider cases where $M_{2} \ll M_{1}$. The above condition to reach synchronization at $a>a_{i} \simeq$ $1.5 R_{1} \simeq 0.4 a_{0}$ gives a constraint of

$$
M_{2} \gtrsim 0.7 \eta M_{\mathrm{env}-\mathrm{t}} \simeq 0.15\left(\frac{\eta}{0.22}\right) M_{\mathrm{env}-\mathrm{t}},
$$

where $M_{\text {env-t }}$ is the giant envelope mass when tidal synchronization takes place. The Darwin instability might occur before or after the primary fills its Roche lobe. We demand that the system enters the Darwin instability when the secondary is outside the primary, but not too close, e.g., $a_{D} \simeq 1.5 R_{1}$. The condition reads

$$
M_{2} \lesssim 0.3\left(\frac{\eta}{0.22}\right)\left(\frac{a_{D}}{1.5 R_{1}}\right)^{-2} M_{\mathrm{env}-\mathrm{D}}
$$

where $M_{\text {env-D }}$ is the giant envelope mass when Darwin instability occurs. Over all, the scenario of ring formation during a Darwin unstable phase reads

$$
0.15\left(\frac{\eta}{0.22}\right) \lesssim \frac{M_{2}}{M_{\text {env }}} \lesssim 0.3\left(\frac{\eta}{0.22}\right),
$$

where we assumed that the envelope mass did not change much between the tidal synchronization and Darwin instability phases.

For the progenitor of SN 1987A condition (3) reads $1.4 M_{\odot} \lesssim M_{2} \lesssim 2.8 M_{\odot}$. In the merger model of Morris \& Podsiadlowski (2009) the secondary mass is $5 M_{\odot}$, and most of the mass is ejected in mid-latitudes, 
not in the equatorial plane. Chevalier \& Soker (1989) required a secondary mass of $M_{2} \simeq 1 M_{\odot}$ to account for the deformation of envelope after the merger. Considering the uncertainties in the above estimates of the binary companion mass, the mass range found by us here can accommodate these models. We emphasize that for the progenitor of SN 1987A we consider a complete rapid merger when the giant core is made out of CO, unlike the incomplete merger discussed by Podsiadlowski et al. (1990).

For a low mass AGB star with an envelope mass of $\sim 0.5-1 M_{\odot}$ near the tip of the AGB, condition (3) reads $M_{2} \simeq 0.08-0.3 M_{\odot}$. This mass range is smaller than the secondary mass range of $0.4-1 M_{\odot}$ mentioned by Miszalski et al. (2013) for the Necklace. We later present our view that the ring in the Necklace was formed in a post-CE event, like a born-again object.

We now turn to examine two processes that can operate more efficiently during a Darwin unstable phase and lead to equatorial mass outflow: the mass loss from the second Lagrangian point $L_{2}$ (section 3 , and the launching of two opposite jets (section 4). The later can work efficiently even if the system is Darwin stable.

\section{EXAMINING EQUATORIAL MASS LOSS}

We consider a binary system where the primary of mass $M_{1}$ losses mass to a secondary star of mass $M_{2}$. The orbit is circular with an orbital separation $a$, and the mass ratio $q \equiv M_{2} / M_{1}<1$. Part or all of this mass is lost through the second Lagrangian point $L_{2}$ located behind the secondary star; the secondary is the accretor in this setting. Livio et al. (1979) already discussed the formation of asymmetrical PNe by mass loss through $L_{2}$. We here repeat those calculations and present them in a way appropriate for our goals. We first consider the angular momentum of the mass leaving through $L_{2}$, and then consider the energy of this mass.

The mass loss process from $L_{2}$ has been discussed in many papers, e.g., Chesneau et al. (2014) for a recent paper on V838 Mon, but in many cases for rotating disks rather than expanding ones (e.g., Millour et al. 2011, Plets et al. 1995). We here discuss only expanding rings/disks that escape from the binary system.

\subsection{Angular momentum at $L_{2}$}

Livio et al. (1979) give the value of the specific angular momentum of mass leaving at $L_{2}$ as

$$
j=\nu \frac{J_{B}}{M_{B}},
$$

where $J_{B}$ and $M_{B}=M_{1}+M_{2}$ are the total angular momentum and mass of the binary system. Based on the results of $\operatorname{Lin}(1977)$, Livio et al. (1979) take $\nu \simeq 1.7\left(M_{B} / \mu\right)$, where $\mu \equiv M_{1} M_{2} /\left(M_{1}+M_{2}\right)$ is the reduced mass of the binary system,. For mass at $L_{2}$ the value is

$$
\nu=\left(\frac{x_{\mathrm{cm}-\mathrm{L}}}{a}\right)^{2} \frac{M_{B}}{\mu}<1.7 \frac{M_{B}}{\mu},
$$

where $x_{\mathrm{cm}-\mathrm{L}}$ is the distance of the center of mass to $L_{2}$, and $a$ is the orbital separation. The reason the value at $L_{2}$ is somewhat smaller than the final value of the angular momentum, by $\sim 10 \%$ for the parameters relevant to us, is that the binary system exerts toque on the mass leaving $L_{2}(\operatorname{Lin} 1977)$.

From angular momentum considerations we can get an expression for the decrease in orbital separation when $(i)$ mass loss occurs only from $L_{2},(i i)$ we neglect the moment of inertia of the primary star, and (iii) 
no mass accretion by the secondary star takes place. Taking a small mass loss such that $q$ does not change much, the equation reads

$$
d J_{B}=j d\left(m_{L}\right)=\nu \frac{J_{B}}{M_{B}} d\left(m_{L}\right),
$$

where $m_{L}$ is the mass lost from $L_{2}$. Taking $m_{L} \ll M_{1}$ such that only the orbital separation $a$ changes in the expression for $J_{B}$, i.e., $d J_{B}=(1 / 2) J_{B} d(\ln a)$, we arrive at the equation

$$
d(\ln a)=2 \nu \frac{d\left(m_{L}\right)}{M_{B}}=3.4 \frac{d\left(m_{L}\right)}{\mu},
$$

where in the second equality we have substitute for $\nu$ from equation (4). For $\left(a_{i}-a\right) / a \ll 1$ the solution is

$$
\frac{a}{a_{i}} \simeq 1-3.4 \frac{m_{L}}{\mu} .
$$

Both accretion of mass by the secondary star and the consideration of the angular momentum of the spinning primary envelope will reduce the orbital separation even faster.

We numerically integrated equation (6) taking into account the primary envelope moment of inertia. As the secondary star spirals-in it spins-up the primary envelope. Synchronization is maintained as long as the system is Darwin-stable. For simplicity we neglect the spin-up of the primary envelope by the secondary star after the system becomes Darwin unstable. In Fig. 1 we present the results for one case with parameters that might fit the progenitor of the Necklace PN. The initial primary mass on the AGB is $1.5 M_{\odot}$ and the secondary mass is $0.4 M_{\odot}$, hence $q=0.267$. The initial orbital separation is $a_{i}=2 \mathrm{AU}$, and the primary radius is $R_{1}=1 \mathrm{AU}$ such that it just fills its Roche lobe, the envelope mass is $M_{\mathrm{env}}=0.9 M_{\odot}$, and the moment of inertia of the giant primary star is $I_{1}=0.22 M_{\mathrm{env}} R_{1}^{2}$. The system reaches Darwin instability when the orbital separation decreases to $1.37 \mathrm{AU}$, as seen by the 'knee' in the lower line. The upper line is according to equation (8), i.e., when the change in the spin angular momentum of the primary star is neglected from the beginning.

Equation (8) and Fig. 1 show that a small mass loss through $L_{2}, m_{L} \lesssim 0.1 M_{2}$ can bring the system to form a CE. This, in principle, can account for the low mass of the rings in the systems discussed in section 1. However, those systems have also bipolar structures, and some more mass might be lost from the system during this phase. As well, the secondary star might accrete some mass. Over all, very little mass will be ejected through $L_{2}$ during the Darwin unstable phase, $m_{L}<0.1 M_{2}$. Although this is compatible with observations of these objects, the energy of the gas at $L_{2}$ poses some problems to this interpretation, as we now show.

\section{2. $\quad$ Energy at $L_{2}$}

We calculated the specific kinetic energy of the mass leaving at $L_{2}$ assuming it has the velocity of the point $L_{2}$. The ratio of the kinetic energy relative to the value of the gravitational energy at $L_{2}$ is presented in Fig. 2. For all value of mass ratio $q$ this ratio is below 1, implying that the mass leaving $L_{2}$ stays bound to the binary system if no further outward acceleration takes place. The final energy will be larger because of the torque exerted on the gas after it leaves $L_{2}$ (Lin 1977). Still, the terminal velocity is smaller than the Keplerian velocity at $L_{2}$.

The expansion velocity of the equatorial ring in the Necklace is $28 \mathrm{~km} \mathrm{~s}^{-1}$, larger than the typical escape velocity from AGB stars and from the typical velocity of AGB winds. In light of the results presented in 


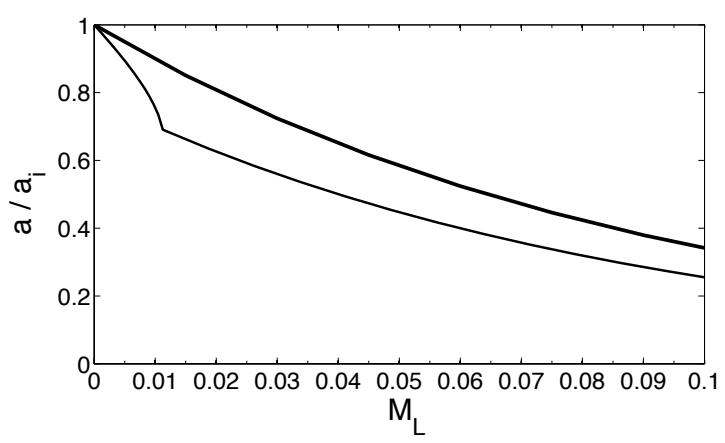

Fig. 1. - The decrease in orbital separation, relative to the initial separation, as function of mass lost from the second lagrangian point $L_{2}$ in units of solar mass. The calculations are for a primary star of $1.5 M_{\odot}$ and a secondary of $0.4 M_{\odot}$, hence $q=0.267$. The initial orbital separation is $a_{i}=2 \mathrm{AU}$, the primary radius $R_{1}=1 \mathrm{AU}$ such that it just fills its Roche lobe, the envelope mass is $M_{\mathrm{env}}=0.9 M_{\odot}$, and the moment of inertia of the giant primary star is $I_{1}=0.22 M_{\text {env }} R_{1}^{2}$. The upper line is for a case where the change in the angular momentum of the spinning primary envelope is neglected, according to equation (8). The lower line is for a case where the primary stellar moment of inertia is taken into account as long as the system is Darwin stable (for $M_{L}<0.011 M_{\odot}$ and $a>1.37 \mathrm{AU}$ for the parameters used here), and the change in the angular momentum of the spinning primary envelope is neglected afterward when the system becomes Darwin unstable (at the break in the lower line: $M_{L}>0.011 M_{\odot}$ ).

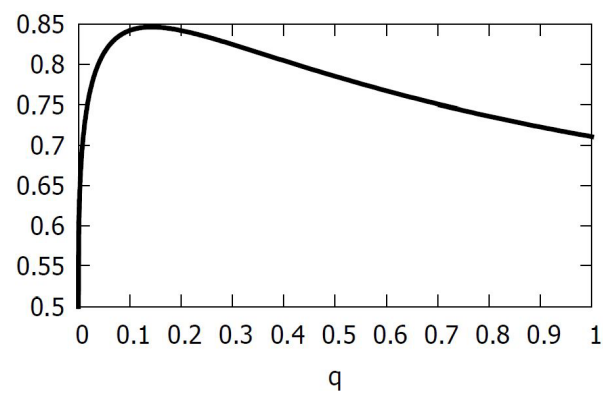

Fig. 2.- The ratio of kinetic energy to the value of the gravitational energy at $L_{2}$. As this ratio is $<1$, if no further acceleration takes place the matter at $L_{2}$ stays bound to the binary system.

Fig. 2 the expansion velocity of the ring in the Necklace calls for one of three explanations. (1) The ring was expelled at the end of the $\mathrm{CE}$ phase, during the so called migration phase where a circumbinary gas is presence (Soker 2013, 2014). Then the orbital separation is $\sim 10 R_{\odot}$, and the escape velocity from the binary system is $\sim 200 \mathrm{~km} \mathrm{~s}^{-1}$. The expansion velocity of the ring is a small fraction of that velocity. This explanation for ring formation is not applicable to the equatorial ring of SN 1987A, as the binary system there did not terminate a $\mathrm{CE}$ phase, and hence we will not explore this possibility here. (2) If the ring was expelled during the AGB phase, it must have experienced an extra acceleration after it left $L_{2}$, much more than the torque by the binary system. (3) The ring was formed when the hot AGB remnant experienced an outburst, e.g., a born-gain AGB process. This explanation holds for the formation of the rings in the 
born-again PNe A30 and A78 that were discussed in section 1 and for novae. We think that the Necklace was formed by option (3), with jets launched by the secondary star.

In principle, the matter leaving $L_{2}$ during an AGB phase could be accelerated by radiation from the giant star or by the thermal pressure of the gas. We see a problem with such a process if a narrow ring is to be formed at later times. The thermal pressure, either from the gas at $L_{2}$ being hot or from radiation pressure, will cause the equatorial gas to expand not only in the equatorial plane, but also in the directions perpendicular to the equatorial plane. It is not clear whether a narrow ring can be formed from such a process. We turn to examine acceleration and focusing of the equatorial outflow by jets.

\section{NUMERICAL SIMULATIONS OF JETS}

Motivated by the presence of bipolar structures in many (or all) of the systems with equatorial rings (section 1), and by the energetic problem discussed in section 3.2. we turn to study the formation of a ring by bipolar jet pairs, following the mechanism proposed by Soker \& Rappaport (2000). In many systems the main bipolar structure was formed before or after the equatorial ring. Non the less, we assume that weaker bipolar jets (or a collimated fast wind; CFW) are launched during the ring formation phase. This can occur either during a Darwin unstable phase or during a stable phase. The same mass that fills the gravitational-potential lobe around the secondary that crosses $L_{2}$, is accreted in part onto the secondary star through an accretion disk. The secondary then is assumed to launch two opposite jets.

\subsection{Numerical setup}

Our simulations are performed by using version 4.0-beta of the FLASH code (Fryxell et al. 2000). The FLASH code is an adaptive-mesh refinement (AMR) modular code used for solving hydrodynamics or magnetohydrodynamics problems. Here we use the unsplit PPM (piecewise-parabolic method) solver of FLASH. We neither include gravity, as velocities are much above the escape speed in the region we simulate, nor radiative cooling, as the interaction region is optically thick. Instead of calculating radiative cooling and radiative transfer, that are too complicated for the flow geometry, we simulate two values of the adiabatic index $\gamma, 4 / 3$ and $5 / 3$. Using lower values of $\gamma<5 / 3$ to mimic radiative cooling is reasonable when kinetic energy is channelled to thermal energy, but not when thermal energy is channelled to kinetic energy. For more details on the numerical settings and the justifications for the parameters employed in this study see Akashi \& Soker (2013).

We employ a full 3D AMR (9 levels; $2^{12}$ cells in each direction) using a Cartesian grid $(x, y, z)$ with outflow boundary conditions at all boundary surfaces. We take the $x-y$ plane with $z=5 \times 10^{15} \mathrm{~cm}$ to be in the equatorial plane of the PN, and we simulate the whole space (the two sides of the equatorial plane).

At $t=0$ we place a spherical dense shell in the region $R_{\text {in }}=10^{14} \mathrm{~cm}<\mathrm{r}<2 \times 10^{14} \mathrm{~cm}=\mathrm{R}_{\text {out }}$, and with a density profile of $\rho_{s}=1.58 \times 10^{-11}\left(r / 10^{14} \mathrm{~cm}\right)^{-2} \mathrm{~g} \mathrm{~cm}^{-3}$, such that the total mass in the shell is $0.1 M_{\odot}$. The gas in the shell has an initial radial velocity of $v_{s}=10 \mathrm{~km} \mathrm{~s}^{-1}$. The shell corresponds to a mass loss episode lasting for $\sim 3 \mathrm{yr}$ and with a constant mass loss rate of $\dot{M}_{s} \simeq 0.03 M_{\odot} \mathrm{yr}^{-1}$. Such an event can be classified as an ILOT, as described in Akashi \& Soker (2013), where more details can be found. The regions outside and inside the dense shell are filled with a lower density spherically-symmetric slow wind having a uniform radial velocity of $v_{\text {wind }}=v_{s}=10 \mathrm{~km} \mathrm{~s}^{-1}$. The slow wind density at $t=0$ is taken to be 
$\rho(t=0)=\dot{M}_{\text {wind }}\left(4 \pi r^{2} v\right)^{-1}$, where $\dot{M}_{\text {wind }}=10^{-5} \mathrm{M}_{\odot} \mathrm{yr}^{-1}$.

The two opposite jets are lunched from the inner $10^{14} \mathrm{~cm}$ region along the $z$-axis and within a half opening angle of $\alpha=50^{\circ}$. By the term 'jets' we refer also to wide outflows, as we simulate here. More generally, we simulate slow-massive-wide (SMW) outflows. Although the jets that are simulated here are not observed (because the medium is optically thick), such wide outflows are commonly observed from active galactic nuclei (e.g., Arav et al. 2013). This, and the success of wide outflows to explain lobes observed in cooling flow clusters (e.g., Sternberg et al. 2007) motivate us to consider wide outflows.

The launching episode lasts for $5 \times 10^{6} \mathrm{~s}=58$ day. The jets' initial velocity is $v_{\text {jet }}=1000 \mathrm{~km} \mathrm{~s}^{-1}$, and the mass loss rate into the two jets together is $\dot{M}_{2 \text { jets }}=0.13 M_{\odot} \mathrm{yr}^{-1}$. The slow wind, dense shell, and the ejected jets start with a temperature of $1000 \mathrm{~K}$. The initial jets' temperature has no influence on the results (as long it is highly supersonic) because the jets rapidly cool due to adiabatic expansion. For numerical reasons a weak slow wind is injected in the sector $\alpha<\theta<90^{\circ}$ (more details are in Akashi \& Soker 2013).

\subsection{Results of main model}

To describe the flow structure of our 3D simulations we will present the density, pressure, and velocity maps in the meridional plane, i.e. a plane through the symmetry axis of the jets, and in planes parallel to the equatorial plane.

We start with the adiabatic index of $\gamma=5 / 3$ case. In Fig. 3 we present the time evolution of the density in the meridional plane at four times. The jets were injected at $r=10^{14} \mathrm{~cm}$ along the $\mathrm{Z}$ axis, with a half opening angle of $50^{\circ}$, and with an initial velocity of $1000 \mathrm{~km} \mathrm{~s}^{-1}$. The jets are active in the time period $t=0$ to $t=58$ days. The regions outside the shells are filled with lower density gas with a radial velocity of $10 \mathrm{~km} \mathrm{~s}^{-1}$. The arrows on the density maps present the velocity direction and magnitude at each point.

In Fig. 4 we present the density maps at two times, 47 days on the left panels and 140 days on the right panels, and in two planes parallel to the equatorial plane. Upper panels are the plane $z=5.05 \times 10^{15} \mathrm{~cm}$, i.e. $\Delta z=0.05 \times 10^{15} \mathrm{~cm}$ from the equatorial plane, and the lower panels are for $z=5.1 \times 10^{15} \mathrm{~cm}$, i.e. $\Delta z=0.1 \times 10^{15} \mathrm{~cm}$. These two planes are marked by horizontal lines in the $t=140$ days panel of Fig. 3

We note the following properties of the flow for the $\gamma=5 / 3$ case. (1) The jets inflate two opposite lobes. Such a general bipolar structure is observed in many PNe and were simulated before (e.g., Akashi \& Soker 2008; Soker et al. 2013). (2) The jets compress some gas toward the equatorial plane (Akashi \& Soker 2008). As seen at 94 and 140 days in figure 3 , a dense and slow equatorial flow is develops. This dense flow will become a ring at late times. (3) The flow becomes Rayleigh-Taylor (RT) unstable (see also Akashi \& Soker 2013). Large RT-tongues develop in the meridional plane, as can be seen on each side of the symmetry axis at times 94 and 140 days in Fig. 3. (4) Other RT unstable regions are seen in the right panels of Fig. 4. These two panels are planes close to the equatorial plane, along which the flow is parallel to the equatorial plane. These many RT-tongues will lead to the formation of dense clumps in the equatorial ring. For this last case we also present in Fig. 5 a three dimensional view of the density structure. The two panels present the density surfaces for different values of the density, as given in the color-bar, and as explained in the caption. Clearly, a clumpy slowly expanding equatorial ring is formed by this flow structure. We note that the exact structure and location of the instabilities are determined also by the numerical grid structure, but their existence is physical.

We turn to the results obtained for the $\gamma=4 / 3$ case, which mimics a more efficient radiate cooling than 


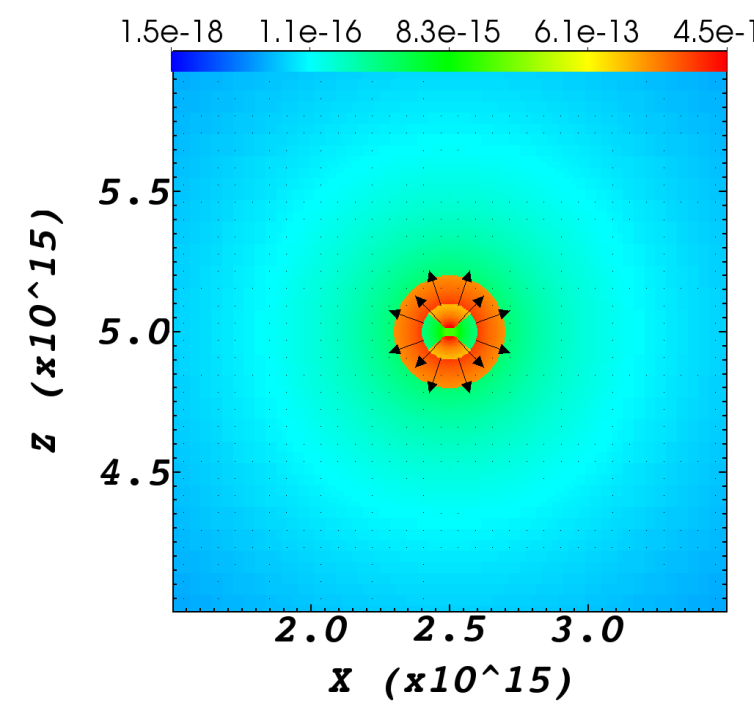

(a) $t=0$ days

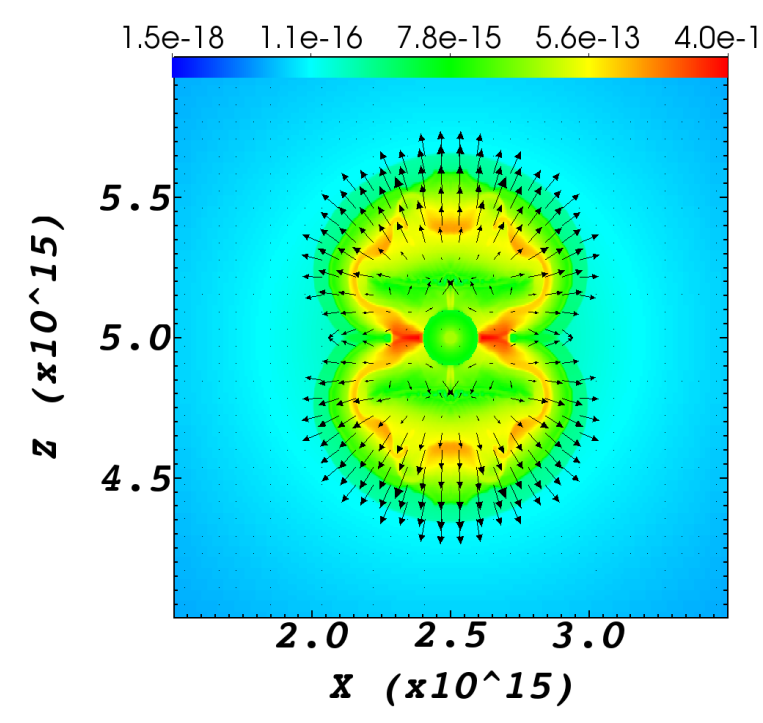

(c) $t=94$ days

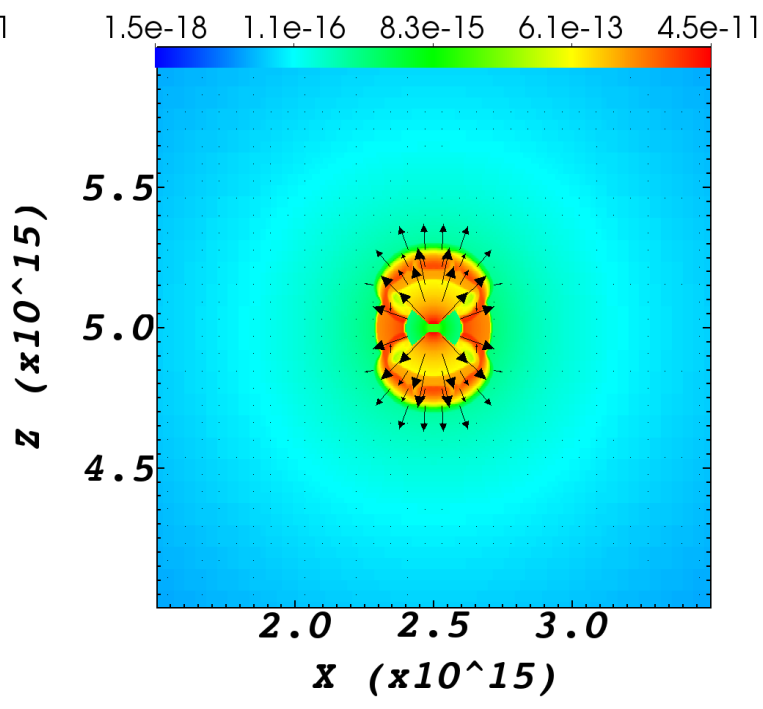

(b) $t=47$ days

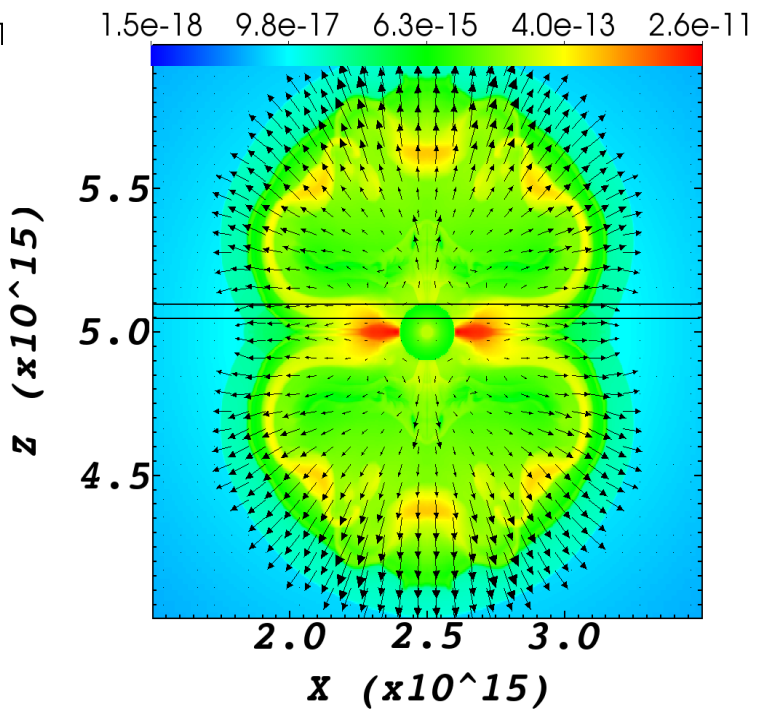

(d) $t=140$ days

Fig. 3.- The density maps in the meridional plane at four times for the $\gamma=5 / 3$ run. Two opposite jets are injected at $r=10^{14} \mathrm{~cm}$ along the $\mathrm{Z}$ axis, with a half opening angle of $50^{\circ}$, with an initial velocity of $1000 \mathrm{~km} \mathrm{~s}^{-1}$, and during the time period $t=0$ to $t=58$ days. The shell, seen in upper left panel, and the low density gas filling the rest of the volume start with a radial velocity of $10 \mathrm{~km} \mathrm{~s}^{-1}$. Arrows depict the flow direction and the velocity magnitude that is relative to the arrow length; the arrows near the center in the first two panels are for $1000 \mathrm{~km} \mathrm{~s}^{-1}$. Density color coding is in units of $\mathrm{g} \mathrm{cm}^{-3}$. The times of panels a, b, c, and d are 0, 47, 94, and 140 days, respectively. Units on the axes are in $\mathrm{cm}$. The two horizontal lines in panel (d) show the planes presented in Fig. 4. Note the development of a dense region in and near the equatorial plane that flows parallel to the equatorial plane. We propose that this equatorial flow turns to a dense ring later on. 


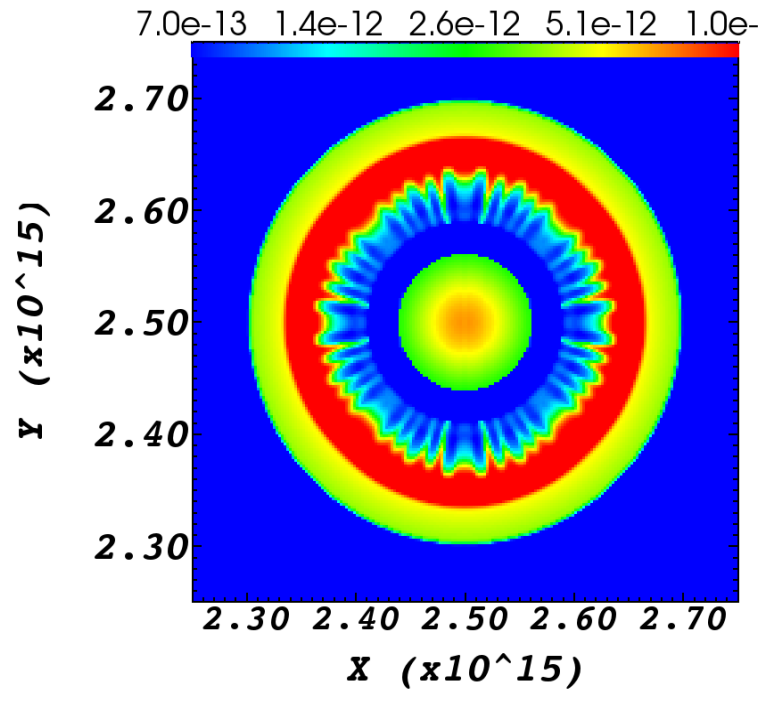

(a) $t=47$ days

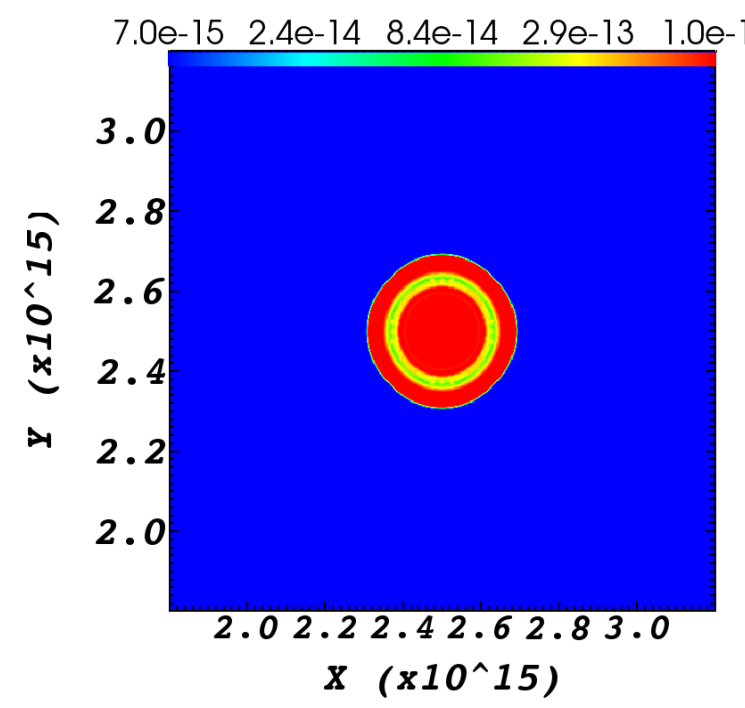

(c) $t=47$ days

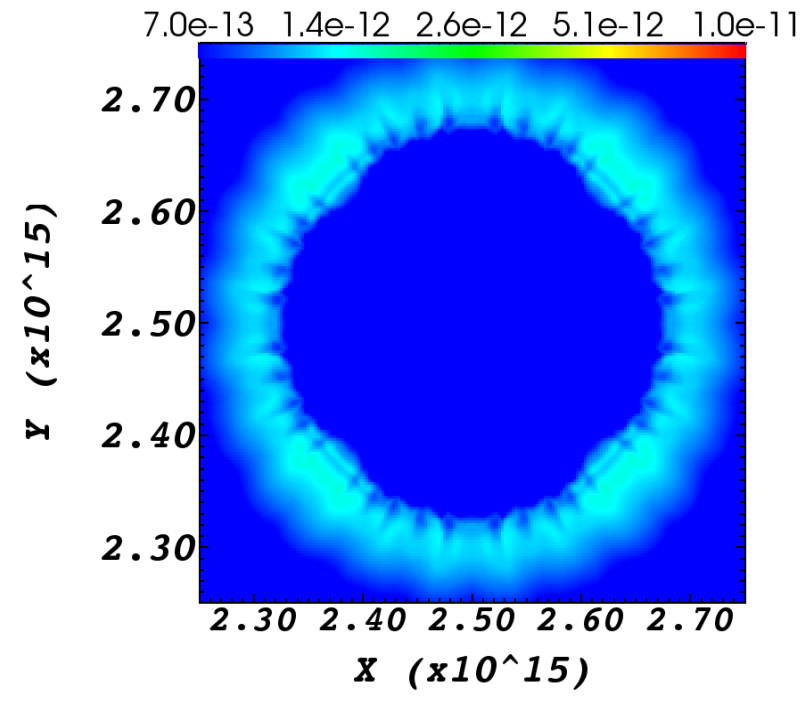

(b) $t=140$ days

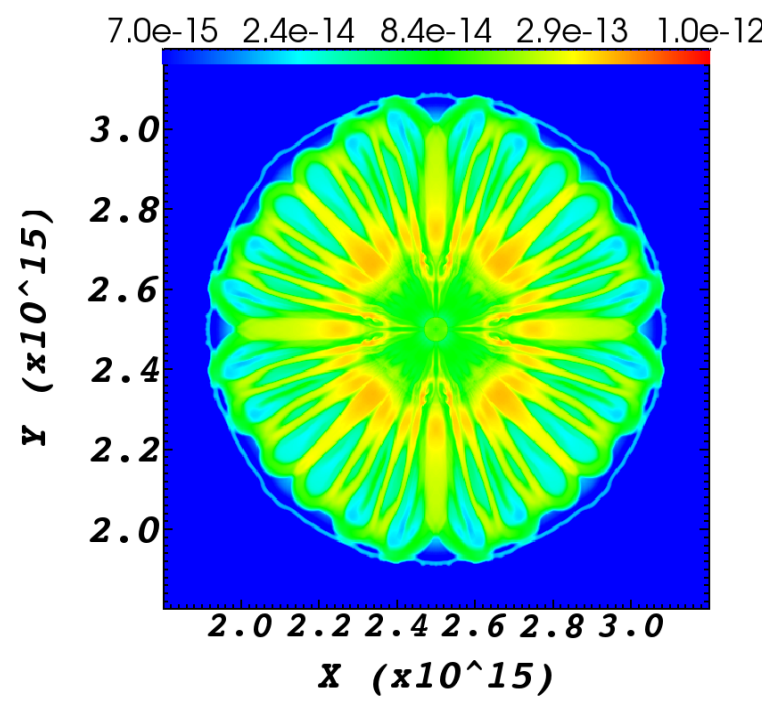

(d) $t=140$ days

Fig. 4. - The density maps at four times of the $\gamma=5 / 3 \mathrm{run}$, and parallel to the equatorial plane. Color coding is in $\mathrm{g} \mathrm{cm}^{-3}$. Left panels are given at times 47 days and right panels at 140 days. Upper panels are the plane $z=5.05 \times 10^{15} \mathrm{~cm}$, i.e. $\Delta z=0.05 \times 10^{15} \mathrm{~cm}$ from the equatorial plane, and the lower panels are for $z=5.1 \times 10^{15} \mathrm{~cm}$, i.e. $\Delta z=0.1 \times 10^{15} \mathrm{~cm}$. Note the development of instabilities at the later times in both planes. These will later form a clumpy ring. The exact structure and location of the instabilities are determined also by the numerical gird, but their existence is physical. Units on the axes are in $\mathrm{cm}$. 


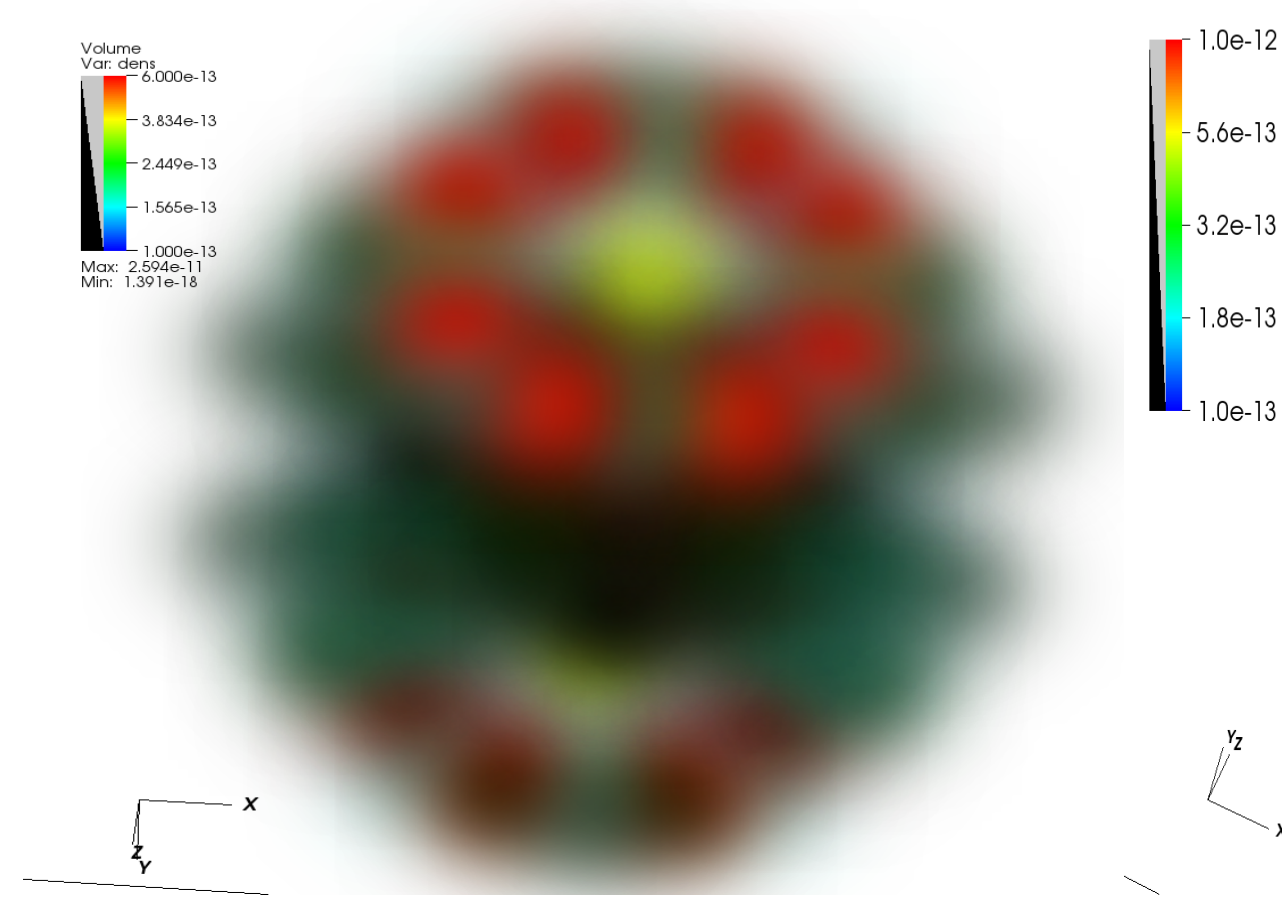

(a) $t=140$ days

(b) $t=140$ days

Fig. 5. - Three dimensional density structure for the $\gamma=5 / 3$ run (main run) presented in figures 3 and 4 . In the left panel, two clumpy rings, the red clumps, are seen in the upper and the lower parts of the nebula. A single ring is seen near the equatorial plane in black. In the right panel, the inner clumpy disk near the center is shown. The inner part of this ring is the densest part of the nebula (the red regions inside the ring). This equatorial ring is the focus of our study. Density color coding is in units of $\mathrm{g} \mathrm{cm}^{-3}$. 
the previous case. In Fig. 6 we present the time evolution of the density in the meridional plane at four times, and in Fig. 7 we present the flow in two planes parallel to the equatorial plane and at two times, like in Fig. 4. We note that there are slight differences between these results to those obtained for the $\gamma=5 / 3$ case. An interesting feature in this run is seen in the lower panels (at 94 and 140 days) of Fig. 6. This is the appearance of two arcs in the most dense region in the equatorial plane. One arc is above and one below the equatorial plane in the figure. Such a split of the denser region can enhance the partition of the equatorial ring into clumps at later times, even clumps that reside entirely only on one side of the equatorial plane. We note the development of instabilities at the later times in both planes presented in Fig. 7, most prominently in panel (b). These will later form a clumpy ring.

To follow the compression of the equatorial flow we show in Fig. 8 the pressure map in the meridional plane at 47 days for the $\gamma=5 / 3$ run. Arrows depict the flow direction but not the magnitude. We clearly see a vortex in the flow that pushes the material toward the equatorial plane. This makes the ring to be denser and enhances the instability.

\section{3. $\quad$ Varying parameters}

To examine the sensitivity of our results we performed numerical simulations with some other initial conditions. In Fig. 9 we show the flow for a case where the initial half-opening angle of the jets is 30 degrees instead of 50 degrees. All other parameters are as in the run presented in Figs. 3 and 4 . We also simulated two cases with a half-opening angle of 50 degrees, but with an initial shell density of third that used in previous runs, for which the results are presented in Fig. 10, and with an initial shell density three times as large that used in previous runs, for which the results are presented in Fig. 11. In all cases the images clearly show the development of an equatorial ring.

\subsection{Summarizing the conditions for ring formation}

A key process for the presently proposed model for the formation of a clumpy equatorial ring is that the CSM gas is compressed toward the equatorial plane by the jets, and that it survives for a long time. For that to occur the following conditions must be met.

(1) Cooling time. Since the initial momentum of each jet is away from the equatorial plane, to exert the force toward that plane, the jet must be shocked and form a hot bubble. For the hot bubble to exert enough pressure its cooling time must be long. Either the radiative emissivity is low, as in Akashi \& Soker (2008), or the photon diffusion time out, $\tau_{\text {diff }}$, is not much shorter than the flow time, $t_{\mathrm{f}}$, as is the case here. The ratio $\tau_{\text {diff }} / t_{\mathrm{f}}$ is given in equation (8) of Akashi \& Soker (2008).

(2) No holes in the CSM. The compression of the CSM toward the equatorial plane must occur before the hot bubble pressure decreases too much. To not lose pressure by gas leakage, there should be no 'holes' in the CSM through which the hot bubble gas can escape.

(3) No hole drilling. Another condition for the bubble pressure not to decrease too rapidly is that the expansion along the polar direction should not be much faster than in other directions. This translates for the jets not to be too narrow. With a half opening angle of $\alpha=30^{\circ}$ we still get a clear ring. The exact limit depends on the other parameters of the jets and the density profile of the CSM. For example, in a case with a little CSM mass along the polar directions and narrow jets, the jets will drill a hole through the CSM, and the hot bubble gas will leak. For the CSM and outflow parameters used here jets with $\alpha \gtrsim 0.1 \pi \simeq 20^{\circ}$ can 


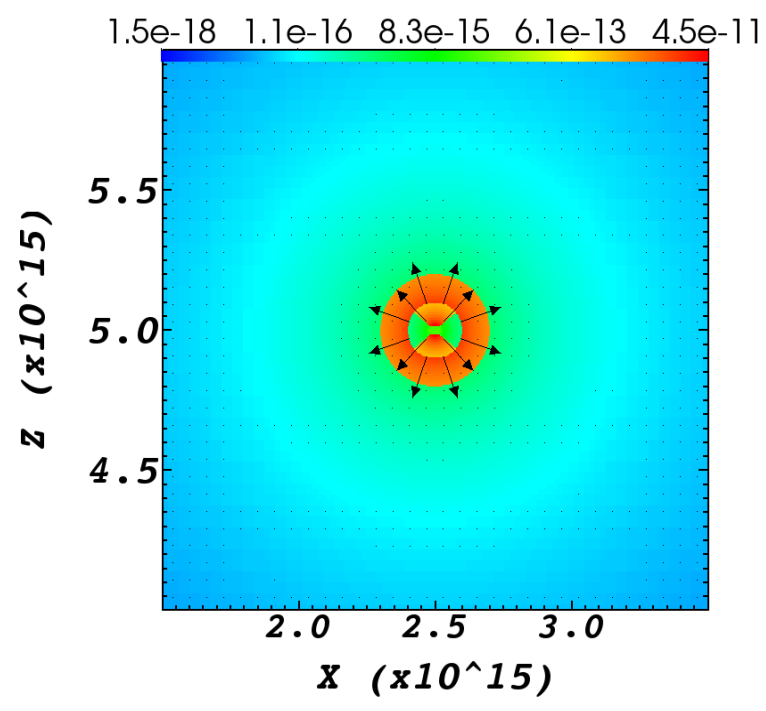

(a) $t=0$ days

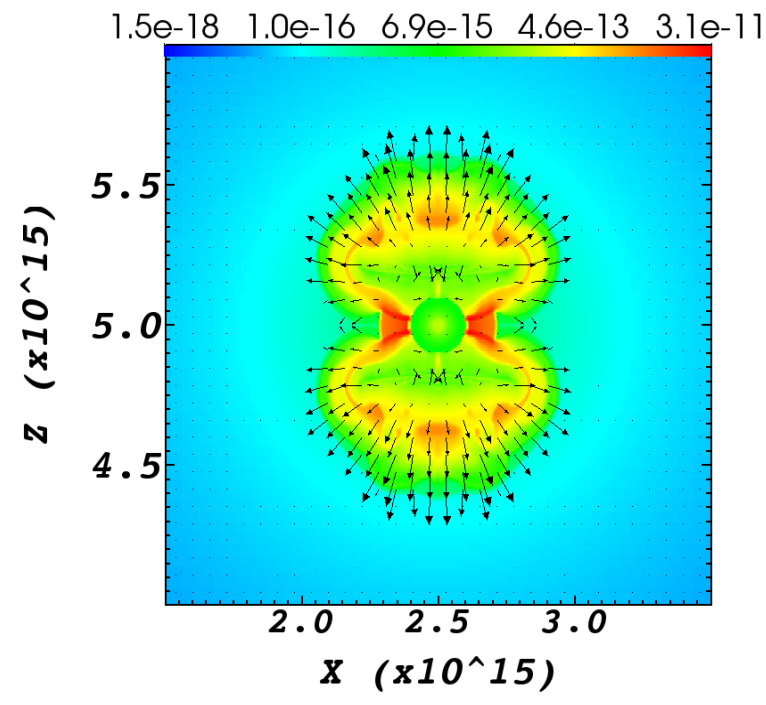

(c) $t=94$ days

Fig. 6. - Like Fig. 3 but for the $\gamma=4 / 3$ case.

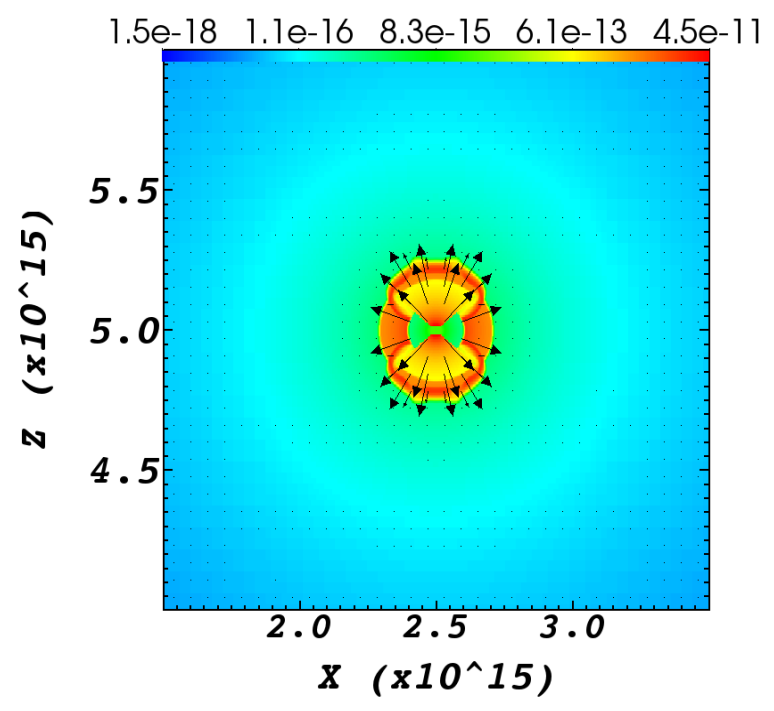

(b) $t=47$ days

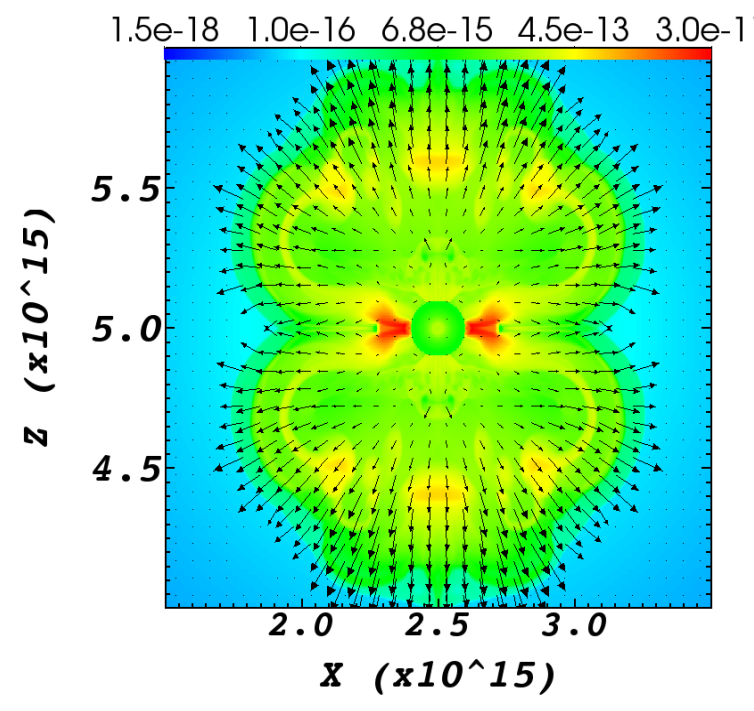

(d) $t=140$ days 


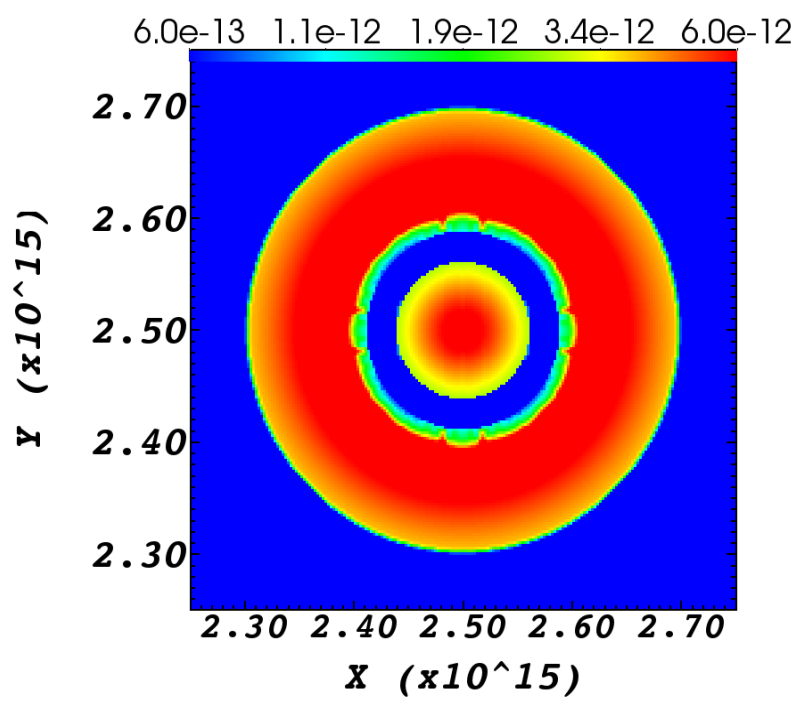

(a) $t=47$ days

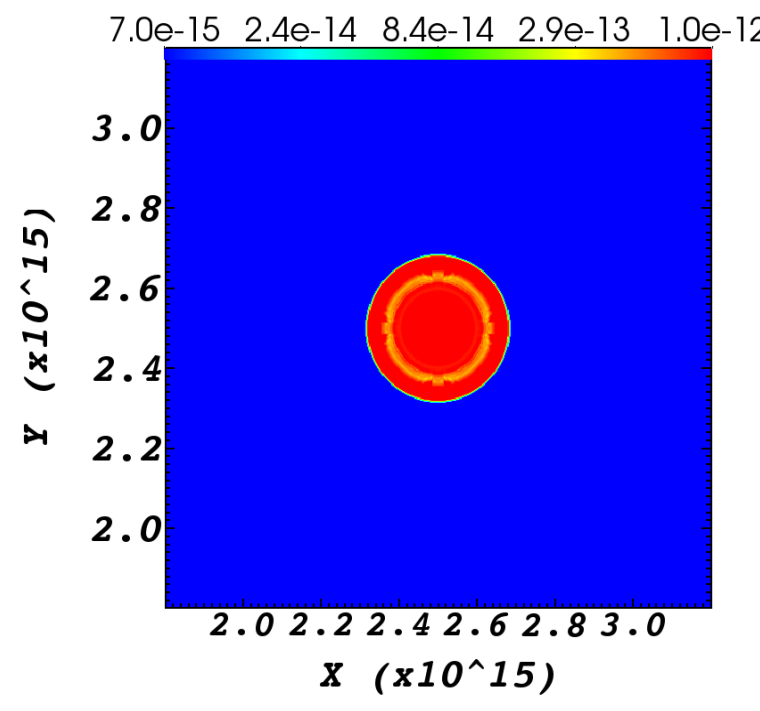

(c) $t=47$ days

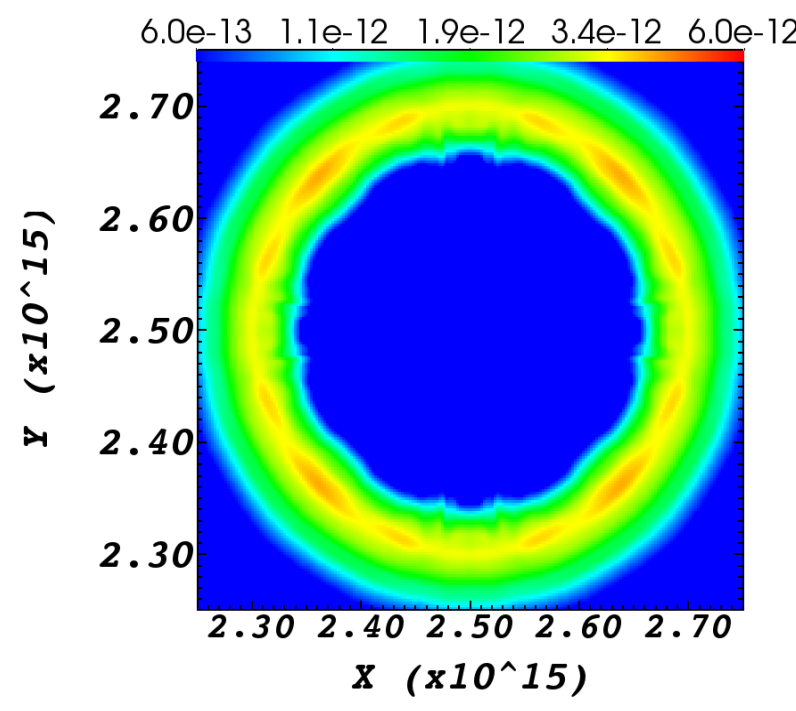

(b) $t=140$ days

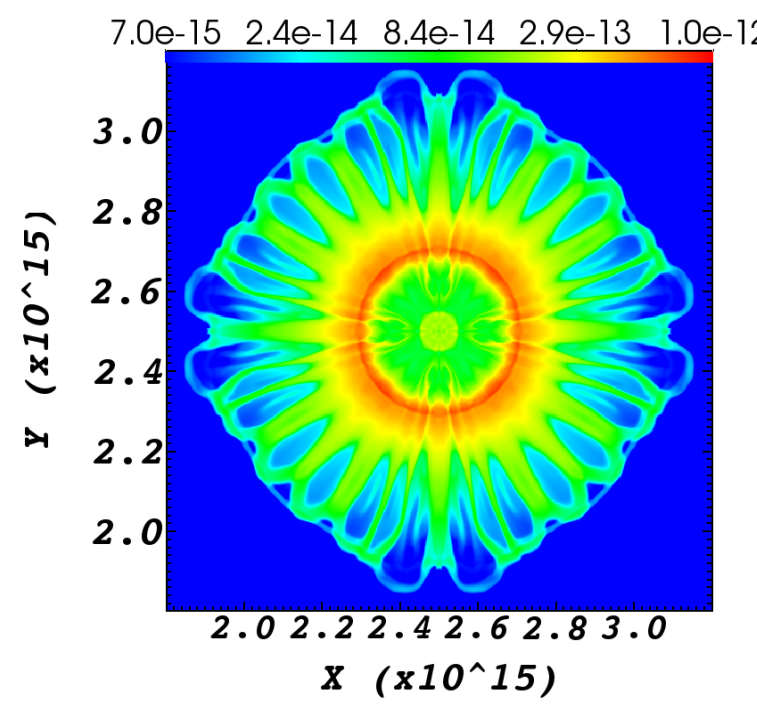

(d) $t=140$ days

Fig. 7.- Like Fig. 4 but for the $\gamma=4 / 3$ case. 


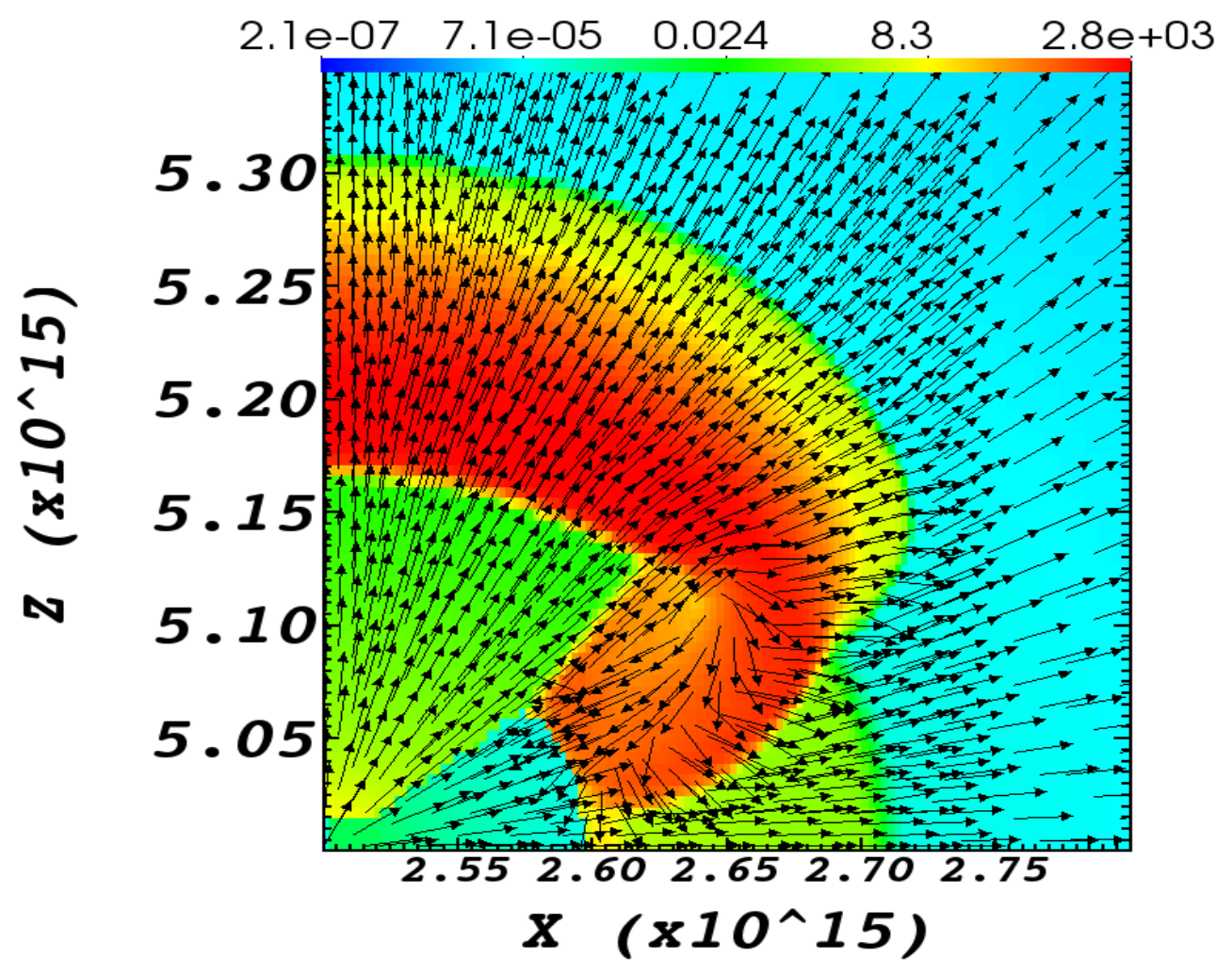

Fig. 8. - The pressure map in the meridional plane at 47 days, for the $\gamma=5 / 3$ run. Arrows depict the flow direction but not the magnitude. Density color coding is in units of $\mathrm{erg} \mathrm{cm}^{-3}$. Units on the axes are in $\mathrm{cm}$. This figure emphasize the formation of a vortex center in this plane on $(2.64,5.11) \times 10^{15} \mathrm{~cm}$. This vortex compresses the equatorial flow. 


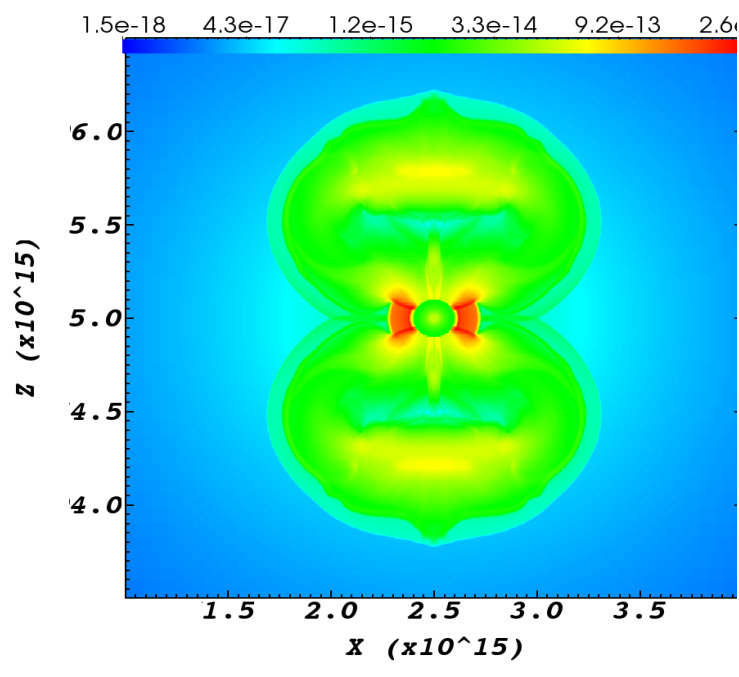

(a) $t=140$ days

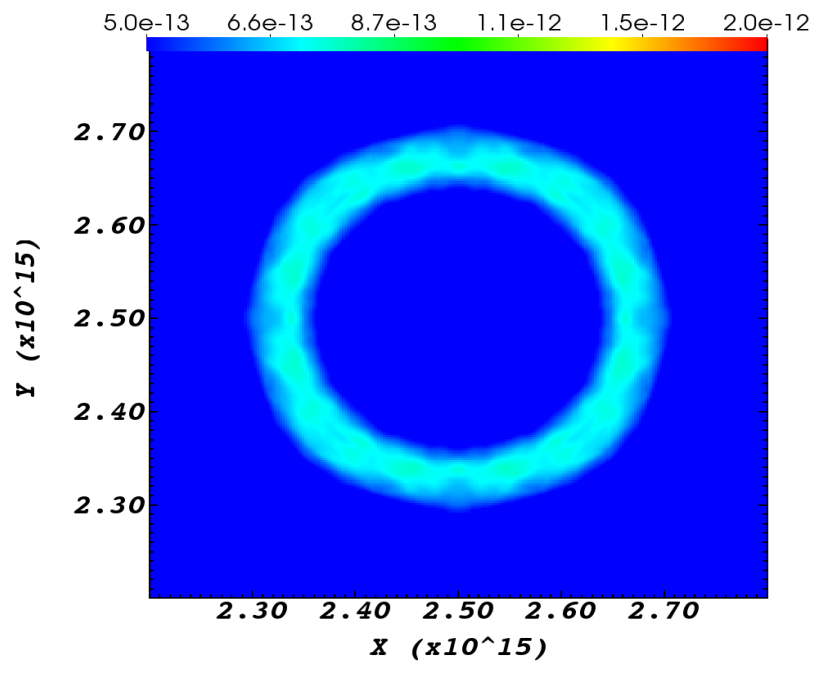

(b) $t=140$ days

Fig. 9.- The density maps in the meridional plane $x z$ (left), and in a plane parallel to the equatorial plane and $\Delta z=0.1 \times 10^{15} \mathrm{~cm}$ above it (right). The half opening angle of the jets is 30 degrees. All other parameters are as in the run presented in Figs. 3 and 4 . Color coding is in $\mathrm{g} \mathrm{cm}^{-3}$ and units on the axes are in $\mathrm{cm}$.

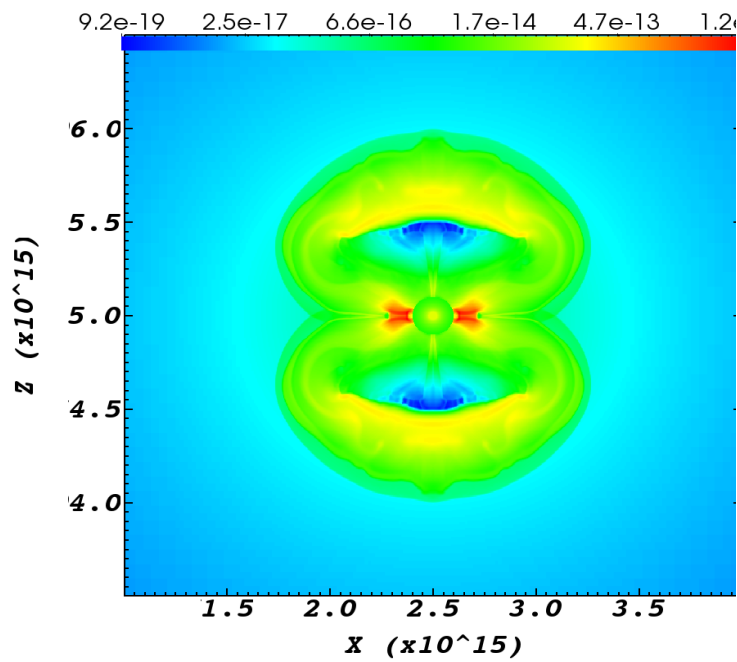

(a) $t=115$ days

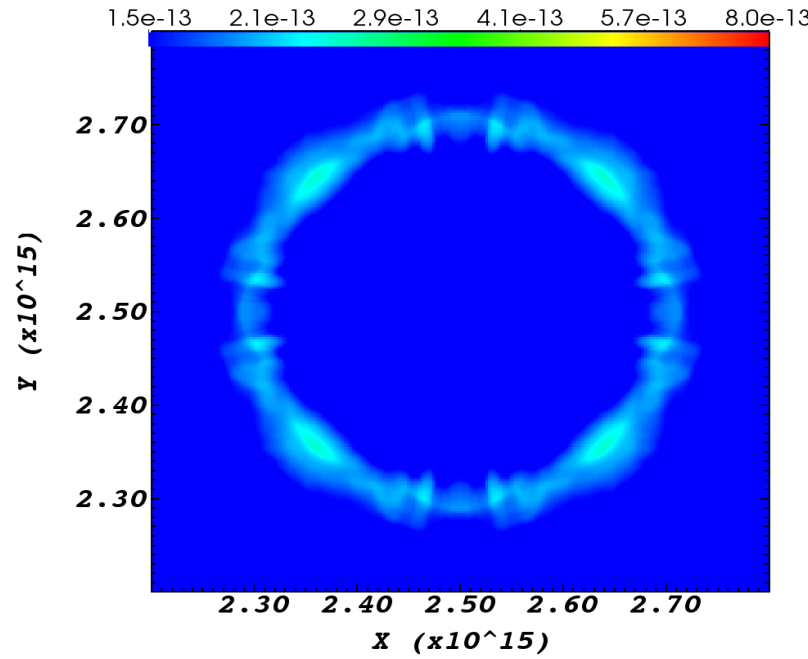

(b) $t=115$ days

Fig. 10.- Like Fig. 9 but the half-opening angle of the jets is 50 degrees and the initial density of the shell is third of that used before. 
lead to a ring formation.

(4) Sufficient energy in the shocked jets. To influence the equatorial gas there should be enough energy in the bubble to compress the CSM near the equatorial plane. In our simulations the total kinetic energy of the jets were about three orders of magnitude more than the energy (thermal and kinetic) of the CSM. However, inspection of the velocity maps show that the fast outflow takes place away from the equatorial plane. Only a small fraction of the jets' energy goes to the compression. In our simulations a clear ring develops early on, even for narrow jets and for dense CSM. We estimate that it is sufficient that the energy in wide-fast jets be more than an order of magnitude of that in the CSM for a ring to form. Narrower jets require more energy for the hot bubble they inflate to form a ring.

(5) No destruction by a late central wind. In some cases, like in SN 1987A and in PNe, after the short interaction, the central star can blow a fast spherical wind. Although the wind is tenuous, it can last for thousands of years. This fast wind can clear the lower density regions of the bipolar bubbles, and leave a large contrast between the equatorial ring and the rest of the nebula. As well, it can lead to the formation of two rings, one above and one below the equatorial plane. It is required that the fast wind does not destroy the ring. Our study of a flow with a spherical fast wind will be published in the near future.

\section{SUMMARY}

We studied the formation of expanding equatorial rings around evolved giant stars (RGB, AGB, and RSG stars). Such rings can reveal themselves when ionized at later times by the central star, either a central star of a PN or an exploding massive star. Based on many similarities between rings around massive stars, that are progenitors of core collapse supernovae (CCSNe), and rings in PNs, we assumed that the ring formation mechanism is similar in all these types of evolved stars (section 1). The main similarity we referred to is the presence of a bipolar nebula in most of these systems.

In section 3 we examined the formation of the ring by mass loss from the second Lagrangian point $\left(L_{2}\right)$ beyond the compact companion to the giant star. This process is most likely to occur when the system is in a Darwin unstable phase (section 2). We found that this process might indeed take place (Livio et al. 1979), but that extra energy must be given to the mass leaving from $L_{2}$. A second problem might be to maintain the flow of gas from $L_{2}$ parallel to the equatorial plane. The gas is close to the binary system, hence hot. It is expected to expand away from the equatorial plane, making the formation of a dense equatorial ring questionable. A third problem might be the formation of a clumpy ring, as the outflow from $L_{2}$ occurs over many orbital periods and the ring is expected to be smooth.

In section 4 we studied the compression of an equatorial ring by bipolar jets, as suggested by Soker \& Rappaport (2000). We found that the process leads to the formation of a dense and clumpy equatorial ring. We therefore suggest that clumpy equatorial rings, such as in the Necklace PN and in AN 1987A, as well as many other similar objects, are formed by this process. In most cases the jets are launched from an accretion disk around a compact companion.

In this first study we did not follow the ring evolution beyond the short time of its formation. In most, or even in all, of the systems with clumpy rings mentioned in section 1, a fast tenuous wind is blown after the intensive mass loss episode. This fast wind might clean the low-density regions, leaving behind only the dense regions, such as the equatorial ring. This might even form polar rings, such as the outer rings in SN 1987A. The later evolution of the ring after the fast tenuous wind has turned on is the subject of a forthcoming paper. 


\section{ACKNOWLEDGEMENT}

This research was supported by the Asher Fund for Space Research at the Technion, and the US-Israel Binational Science Foundation.

\section{REFERENCES}

Akashi, M., \& Soker, N. 2008, NewA, 13, 157

Akashi, M., \& Soker, N. 2013, MNRAS, 436, 1961

Arav, N., Borguet, B., Chamberlain, C., Edmonds, D., \& Danforth, C. 2013, MNRAS, 436, 3286

Bond, H. E., Bedin, L. R., Bonanos, A. Z., Humphreys, R. M., Monard, L. A. G. B., Prieto, J. L., \& Walter, F. M. 2009, ApJ, 695, L154

Boumis, P., \& Meaburn, J. 2013, MNRAS, 430, 3397

Brandner, W., Chu, Y.-H., Eisenhauer, F., Grebel, E. K., \& Points, S. D. 1997, ApJ, 489, L153

Bujarrabal, V., Alcolea, J., Van Winckel, H., Santander-García, M., \& Castro-Carrizo, A. 2013a, A\&A, 557, A104

Bujarrabal, V., Castro-Carrizo, A., Alcolea, J., Van Winckel, H., Sanchez Contreras, C., Santander-Garca, M., Neri, R., \& Lucas, R. 2013b, A\&A, 557, L11

Chesneau, O., Millour, F., De Marco, O., et al. 2014, A\&A, 569, L3

Chevalier, R. A., \& Soker, N. 1989, ApJ, 341, 867

Chita, S. M., Langer, N., van Marle, A. J., García-Segura, G., \& Heger, A. 2008, A\&A, 488, L37

Chomiuk, L., Linford, J. D., Yang, J., et al. 2014, Nature, 514, 339

Cohen, M., Van Winckel, H., Bond, H. E., \& Gull, T. R. 2004, AJ, 127, 2362

Corradi, R. L. M., Sabin, L., Miszalski, B., et al. 2011, MNRAS, 410, 1349

Fang, X., Guerrero, M. A., Marquez-Lugo, R. A., et al. 2014, ApJ, 797, 100

Fryxell, B., Olson, K., Ricker, P., et al. 2000, ApJS, 131, 273

Jura, M., Balm, S. P., \& Kahane, C. 1995, ApJ, 453, 721

Kashi, A., Frankowski, A., \& Soker, N. 2010, ApJ, 709, L11

Kashi, A., \& Soker, N. 2010b, (arXiv:1011.1222)

Kasliwal, M. M., et al. 2011, ApJ, 730, 134

Larsson, J., Fransson, C., Kjaer, K., et al. 2013, ApJ, 768, 89

Lin, D. N. C. 1977, MNRAS, 179, 265

Livio, M., Salzman, J., \& Shaviv, G. 1979, MNRAS, 188, 1

Mattila, S., Lundqvist, P., Gröningsson, P., Meikle, P., Stathakis, R., Fransson, C., \& Cannon, R. 2010, ApJ, 717, 1140

Millour, F., Meilland, A., Chesneau, O., Stee, Ph., Kanaan, S., Petrov, R., Mourard, D., \& Kraus, S. 2011, A\&A, 526, A107

Miszalski, B., Boffin, H. M. J., \& Corradi, R. L. M. 2013, MNRAS, 428, L39

Morris, T., \& Podsiadlowski, P. 2009, MNRAS, 399, 515

Paxton, B., Bildsten, L., Dotter, A., et al. 2011, ApJS, 192, 3

Plait, P. C., Lundqvist, P., Chevalier, R. A., \& Kirshner, R. P. 1995, ApJ, 439, 730

Plets, H., Waelkens, C., \& Trams, N. R. 1995, A\&A, 293, 363

Podsiadlowski, P., Joss, P. C., \& Rappaport, S. 1990, A\&A, 227, L9

Potter, T.M., Staveley-Smith, L., Reville, B., Ng, C.-Y., Bicknell, G. V., Sutherland, R. S., \& Wagner, A. Y. 2014, ApJ, 794, 174

Prieto, J. L., Sellgren, K., Thompson, T. A., \& Kochanek, C. S. 2009, ApJ, 705, 1425

Retter, A. 2004, ApJ, 615, L125

Rubio, G., Vázquez, R., Ramos-Larios, G., Guerrero, M. A., Olguin, L., Guillen, P. F., \& Mata, H. 2015, MNRAS, 446, 1931 
Smith, N. 2007, AJ, 133, 1034

Smith, N., Arnett, W. D., Bally, J., Ginsburg, A., \& Filippenko, A. V. 2013, MNRAS, 429, 1324

Soker, N. 1996, ApJ, 460, L53

Soker, N. 2013, NewA, 18, 18

Soker, N. 2014, arXiv:1404.5234

Soker, N. 2007, ApJ, 661, 490

Soker, N., Akashi M., Gilkis A., Hillel S., Papish O., Refaelovich M., \& Tsebrenko D. 2012, AN, 334, 402

Soker, N., \& Kashi, A. 2012, ApJ, 746, 100

Soker, N., \& Rappaport, S. 2000, ApJ, 538, 241

Sternberg, A., Pizzolato, F., \& Soker, N. 2007, ApJ, 656, L5

Sugerman, B. E. K., Crotts, A. P. S., Kunkel, W. E., Heathcote, S. R., \& Lawrence, S. S. 2005, ApJS, 159, 60

Taylor, W. D., Evans, C. J., Simón-Díaz, S., Sana, H., Langer, N., Smith, N., \& Smartt, S. J. 2014, MNRAS, 442, 1483

Tocknell, J., De Marco, O., \& Wardle, M. 2014, MNRAS, 439, 2014

Tylenda, R., Kaminski, T., Udalski, A., et al. 2013, A\&A, 555, AA16

van Winckel, H. 2003, ARA\&A, 41, 391

Zanardo, G., Staveley-Smith, L., Ng, C.-Y., et al. 2013, ApJ, 767, 98 


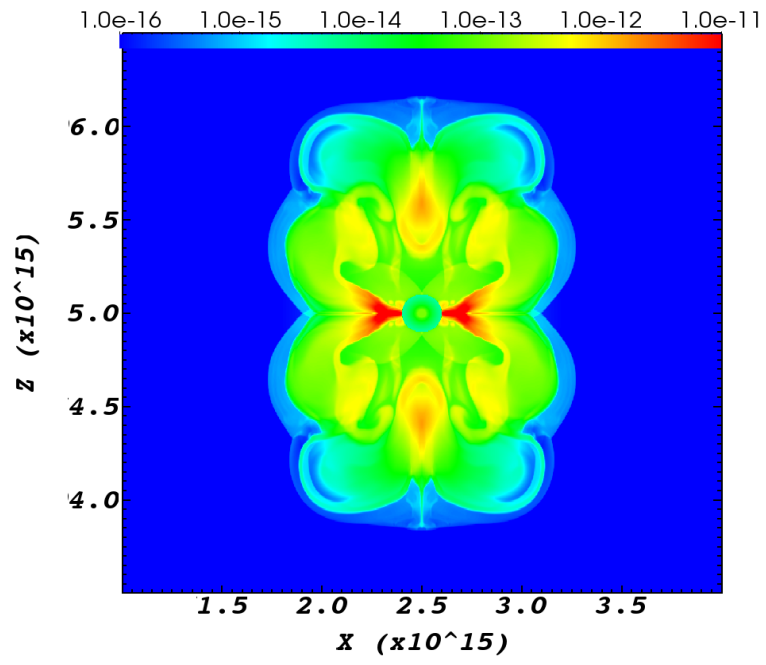

(a) $t=195$ days

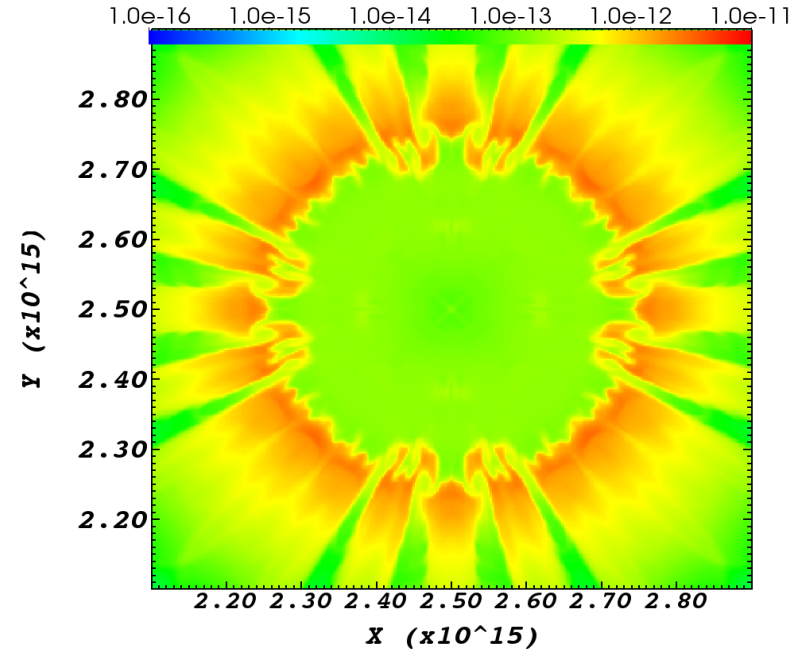

(b) $t=195$ days

Fig. 11. - Like Fig. 9 but the half-opening angle of the jets is 50 degrees and the initial density of the shell is three times of that used before. 